\title{
Cine MRI of swallowing in patients with advanced oral or oropharyngeal carcinoma: a feasibility study
}

\author{
Anne Marijn Kreeft • Coen R. N. Rasch • \\ Sara H. Muller · Frank A. Pameijer · Eeke Hallo • \\ Alfons J. M. Balm
}

Received: 27 August 2011 / Accepted: 21 November 2011 / Published online: 5 January 2012

(C) The Author(s) 2012. This article is published with open access at Springerlink.com

\begin{abstract}
Treatment of oral and oropharyngeal cancer may cause dysphagia. Purpose is to examine whether cine magnetic resonance imaging (MRI) yields additional information compared to standard examination in the evaluation of posttreatment dysphagia and mobility of oral and oropharyngeal structures. Thirty-four cine MRIs were made in 23 patients with advanced oral and oropharyngeal cancer, consisting of an MR image every $800 \mathrm{~ms}$ during swallowing which is compared to videofluoroscopy and quality of life questionnaires. A scoring system was applied to assess mobility on
\end{abstract}

A. M. Kreeft · A. J. M. Balm

Department of Head and Neck Oncology and Surgery,

The Netherlands Cancer Institute, Antoni van Leeuwenhoek

Hospital, Plesmanlaan 121, 1066 CX Amsterdam,

The Netherlands

C. R. N. Rasch ( $\varangle)$

Department of Radiotherapy, The Netherlands Cancer Institute, Antoni van Leeuwenhoek Hospital, Plesmanlaan 121,

1066 CX Amsterdam, The Netherlands

e-mail: c.rasch@nki.nl

\section{S. H. Muller}

Department of Clinical Physics, The Netherlands Cancer Institute, Antoni van Leeuwenhoek Hospital, Plesmanlaan 121,

1066 CX Amsterdam, The Netherlands

\section{F. A. Pameijer}

Department of Radiology, University Medical Center Utrecht, Heidelberglaan 100, 3584 CX Utrecht, The Netherlands

E. Hallo

The Netherlands Cancer Institute, Antoni van Leeuwenhoek Hospital, Plesmanlaan 121, 1066 CX Amsterdam,

The Netherlands

\section{A. J. M. Balm}

Department of Otorhinolaryngology, Academic Medical Center, Meibergdreef 9, 1105 AZ Amsterdam, The Netherlands cine MR and videofluoroscopy leading to a score ranging from 9 to 17. Cine MRI of the swallowing in a midsagittal plane visualized the tumor (if located in the same plane), important anatomic structures and surgical reconstructions. Posttreatment mobility on cine MRI and videofluoroscopy was significantly diminished compared to pretreatment, mean pretreatment cine MRI score was 10.8 and posttreatment $12.4(p=0.017)$. Impaired mobility on cine MRI was significantly correlated to more swallowing problems (Spearman's correlation coefficient $0.73, p=0.04$ ), on videofluoroscopy not. Cine MRI is a promising new technique as an adjunct to standard examinations for evaluation of swallowing in patients with oral and oropharyngeal cancer. Cine MRI directly visualizes the dynamics of swallowing and allows evaluation of pre- and posttreatment differences. Abnormal findings are significantly correlated with subjective swallowing complaints of patients.

Keywords Swallowing - Cine MRI - Oral and oropharyngeal cancer · Oral function · Quality of life

\section{Background}

The oral cavity and oropharynx are mobile structures with complex movements during swallowing and speech. Treatment of cancer in this region may cause serious function losses with regard to speech and swallowing, especially in advanced cancer stages [1,2].

Two treatment modalities are available for advanced oral and oropharyngeal cancers; surgical therapy, frequently combined with postoperative radiotherapy (RT) and organ sparing therapy consisting of concurrent chemo- and radiotherapy [3]. Both treatment regimes result in comparable survival rates [4-7] and have their particular functional 
consequences. Function losses after surgery depend on the amount and location of the tissue that is removed and the reconstruction techniques. Soft tissue defects are reconstructed using free or pedicled flaps, filling up the defect, but resulting in a nonfunctional mass. Xerostomia and fibrosis of oral and oropharyngeal tissues play a role in development of swallowing problems after RT, depending on radiation dose and volume [8].

Nowadays, oral function is assessed by videofluoroscopy. Mobility and function of involved structures remain difficult to evaluate. A more accurate functional imaging modality, visualizing both tumor extension and mobility may yield additional information.

Earlier reports describe the use of cine Magnetic Resonance Imaging (MRI) to visualize the dynamic structures of the oral cavity and oropharynx. Hartl et al. [9] applied cine MRI for evaluation of anatomical and physiological characteristics, consisting of $5 \times 15$ different images made during swallowing. They showed that oral preparatory, oral and oropharyngeal phases of deglutition were visible and that spatial resolution allowed for anatomical measurements of laryngeal elevation, oropharyngeal diameter, and tongue base and soft palate displacement. Furthermore, in a following study, they [10] applied this technique to evaluate 6 patients with persistent dysphagia after tumor treatment to analyze the morphology and mobility of the oral, oropharyngeal and laryngeal structures. This yielded further information on the cause of dysphagia in five of six cases. Kitano et al. [11] described cine MRI in 12 patients with dysphagia after surgery and postoperative RT for oral cancer and successfully analyzed swallowing ability. Cine MRI may give clinicians additional knowledge on the impact of cancer treatment on structures in the oral cavity and oropharynx. Cine MRI may also enhance insight in tumor location and treatment induced immobility of surrounding structures, which might help in making the difficult choice between the two treatment regimes.

The first purpose of this study was to establish the feasibility of cine MRI of swallowing in patients with advanced oral and oropharyngeal cancer for evaluation of tumor extension, and evaluation of visibility and mobility of important structures in the oral cavity and oropharynx, including various tongue muscles. Secondary purposes were to analyze the additional value of cine MRI compared to standard examinations, visualization of pre- and posttreatment differences in mobility and evaluation of the clinical consequences for quality of life of deviations on cine MRI.

\section{Patients}

From December 2008 until July 2009, all patients with newly diagnosed advanced (T2-4) squamous cell carci- noma in the oral cavity and oropharynx that were planned for curative therapy (surgery or concurrent chemoradiation) were subsequently included. Exclusion criteria were contraindications for MRI, tumor histology other than squamous cell carcinoma and palliative therapy.

\section{Methods}

\section{Cine MRI parameters}

Cine MRI images were obtained using a 3-T MRI unit (Achieva, Philips, Best, Netherlands). Patients were in a standard supine position. One 15-mm median sagittal slice, centered on the vallecular region comprising the oral and pharyngeal cavities from the lips to the cervical esophagus, was obtained from each acquisition. A mildly T2 weighted single-shot turbo spin echo technique was used with a repetition time of $800 \mathrm{~ms}$ (per acquisition slice), and the following parameters: echo time $44 \mathrm{~ms}$, field of view $230 \times 132 \mathrm{~mm}^{2}$, slice thickness $5 \mathrm{~mm}$, pixels size acquisition $1.8 \times 2.0 \mathrm{~mm}$, reconstruction $0.6 \mathrm{~mm}^{2}$, NSA $=1$, flip angle 90 , linear profile order, refocusing $120^{\circ}$, sense factor 2, half scan 0.6, TSE Factor 37 . One series of 25 acquisitions in the median sagittal plane was obtained for each patient. The subjects were instructed to repeatedly swallow in a natural manner and at a comfortable rate. No bolus (water or other) was administered because of risk of aspiration $[9,10]$.

For the first part of the study, only a pretreatment MRI was made. In the second part of the study a pretreatment cine MRI and a standard videofluoroscopy was made and the patient was asked to fill in the quality of life questionnaire. This protocol was repeated approximately 10 weeks after the last radiotherapy or day of surgery.

\section{Cine MRI scoring}

Cine MRI images studies consisted of 25 images in different stages of swallowing. Quality of cine MRI was evaluated based on the sharpness of the images and the visibility of the most important landmarks in oral and oropharyngeal swallowing.

An immobility score was defined for subjective evaluation of the cine MRI, based both on mobility of the particular structures, the anterior tongue, the base of tongue, the posterior pharyngeal wall, the palate and the floor of mouth, (see Table 1). Mobility of these structures was subjectively assessed and scored as: "1" (normal), "2" (somewhat decreased) and " 3 " (decreased/immobile). Contact between anterior tongue- palate, BOT- posterior pharyngeal wall, BOT- soft palate and soft palate- posterior pharyngeal wall was evaluated and scored as: "1" (visible contact/normal), "2" (no visible contact/abnormal). Adding the scores of 
Table 1 Immobility score for cine MRI and videofluoroscopy

\begin{tabular}{lllll}
\hline Mobility $^{\mathrm{a}}$ & Anterior tongue & BOT & Soft palate & PPW \\
Contact $^{\mathrm{b}}$ & Soft palate-PPW & BOT-PPW & BOT-soft palate & Anterior tongue-palate \\
\hline
\end{tabular}

$B O T$ base of tongue, FOM floor of mouth, $P P W$ posterior pharyngeal wall

${ }^{a}$ Mobility was subjectively scored by two independent evaluators, using the following scores: 1 normal, 2 somewhat decreased, 3 decreased

${ }^{\mathrm{b}}$ Contact between structures was subjectively scored by two independent evaluaters, using the following scores: 1 visible contact, 2 no visible contact. Items that could not be visualised sufficiently to evaluate contact or mobility, were given a normal score (=1), see Methods section for more information

these 9 items, the immobility score varies in theory between "9" (normal/mobile) and " 23 " (completely abnor-

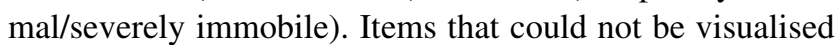
sufficiently to evaluate contact or mobility, were given a normal score (1), and the number of items that could not be evaluated was counted (ranging from 0 to 9). Discrimination and mobility of genioglossus and geniohyoid muscle and intrinsic tongue muscles was additionally evaluated, though not included in above mentioned cine MRI immobility score as it would overestimate the score to include both the structure and the underlying muscle.

Cine MRI images were rated on quality and immobility subjectively by one author (AK). A quarter of all cine MRI evaluations were repeated by the same investigator again and by an experienced head and neck radiologist (FP) to calculate the intra- and interobserver reliability score. In case of disagreement, the study was re-evaluated by the two reviewers and a conclusion was reached in consensus.

\section{Videofluoroscopy}

All videofluoroscopies were evaluated according to the same criteria as cine MRI and immobility scores were calculated in the same way for videofluoroscopy (see Table 1) by one author (AK) and re-evaluated by an experienced speech therapist $(\mathrm{EH})$.

\section{EORTC-H\&N 35 questionnaire Quality of life questionnaire and dysphagia score}

The quality of life questionnaire was based on the EORTCH\&N 35 [12]. Questions from the EORTC- H\&N 35 questionnaire were used about oral transport, swallowing of solid food, soft/grounded food and fluids, whether several swallows are necessary and whether it is necessary to drink whilst eating. Correlation of the 10 questions in the questionnaire regarding swallowing symptoms was checked by reliability analysis on SPSS, using Cronbach's alpha test for assessing internal consistency. For scales, which are used as research tools to compare groups, alpha values of $0.7-0.8$ are regarded as satisfactory. For the clinical application, much higher values are needed, as the value of the scale is for an individual of interest and then the minimum alpha value is 0.90 , and desirable is 0.95 [13]. After correlation analysis, the sum of 10 questions regarding swallowing resulted in a dysphagia score ranging from 10-37; 10 meaning normal swallowing and 37 meaning severe problems with swallowing or severe dysphagia.

Analysis

Spearman's rank correlation coefficient between the dysphagia score and immobility scores of videofluoroscopy and cine MRI was calculated using SPSS. Correlation coefficients range from +1 to $-1,+1$ or -1 occurs when each of the variables is a perfect monotone function of the other.

Differences in immobility scores on cine MRI and videofluoroscopy pre- and posttreatment were calculated using nonparametric testing with Wilcoxon Signed Rank test. Differences in results between T-stages were evaluated using the Mann-Whitney $U$ test, as groups were unequally balanced.

\section{Results}

In total 23 patients were included and 34 cine MRIs were made and evaluated. The first four patients only underwent pretreatment cine MRI as we first wanted to establish that image quality of the cine MRI was satisfactory. Four patients were excluded after the first cine MRI because of local recurrence $(n=1)$, distant metastases and palliative therapy $(n=2)$ and tumor histology other than squamous cell carcinoma ( $n=1$, lymphoma). One patient died and three patients refused to undergo the second cine MRI because of the extra visit to the hospital $(n=1)$ and claustrophobia $(n=2)$. A total of eleven patients were available for analysis in the second part of the study with data of a pre- en posttreatment cine MRI. For these 11 patients also videofluoroscopy and quality of life questionnaires were planned pre- and posttreatment.

\section{Feasibility of cine-MRI for imaging of swallowing}

Thirty-four studies, 23 pretreatment and 11 posttreatment, were available for evaluation of the first purpose, namely evaluation of the visibility of structures and its mobility and 
Fig. 1 Cine MRI of a patient with a $\mathrm{T} 4 \mathrm{aN} 2 \mathrm{c}$ tongue carcinoma. 3 of the 25 Cine MRI images showing different phases of swallowing: red immobile tumor, blue mobile base of tongue

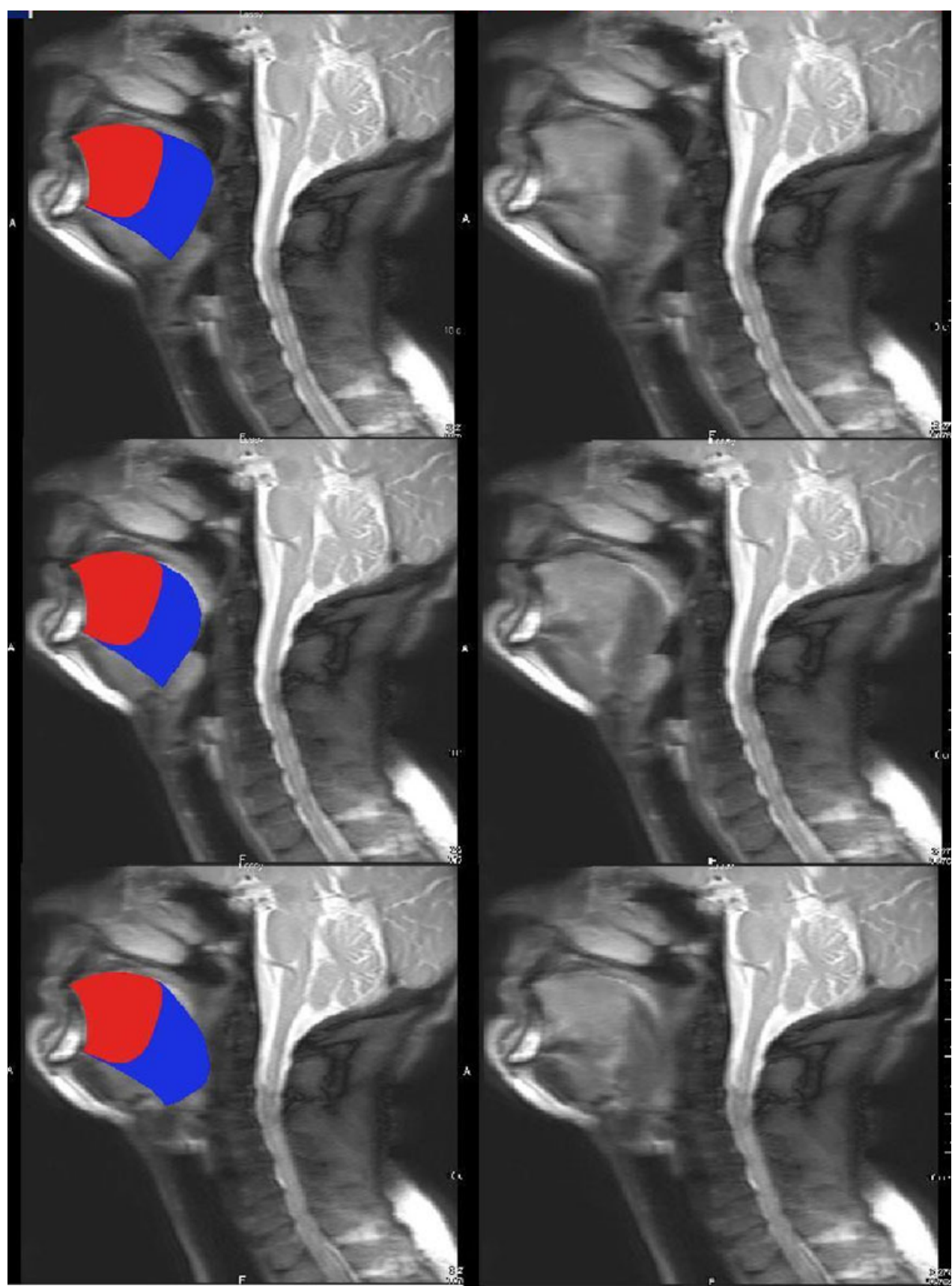

the visibility of the tumor and evaluation of the quality of cine MR images, see Fig. 1 and the movies in the online version of the journal. Twenty were considered of good quality, ten of moderate quality and 4 of low quality, because not all structures were visible $(n=2)$ and images were not sharp $(n=1)$ or all in the same swallowing phase $(n=1)$. Images were blurry due to motion artifacts caused by a fast swallowing action.

In the pretreatment cine MRIs $(n=23)$, all midline tumors were visible $(n=18)$, because the slice was taken in the midsagittal plane. In five more laterally located tumors, the tumor could not be identified.
Important structures for the swallowing movement, such as the anterior tongue, base of tongue (BOT), the soft palate, posterior pharyngeal wall, floor of mouth and epiglottis were all visible on cine MRI; see Fig. 1 and see video files in the online version of the journal. It was also possible to evaluate mobility of the base of tongue, anterior tongue, soft palate floor of mouth, and often also of the pharyngeal back wall. Differences in mobility between these structures were observed; see Fig. 1. In 34 cine MRIs, these structures were all distinguished, except for the epiglottis and posterior pharyngeal wall that were not visualized in 2 cine MRIs that were of low quality. The evaluation of the cine 


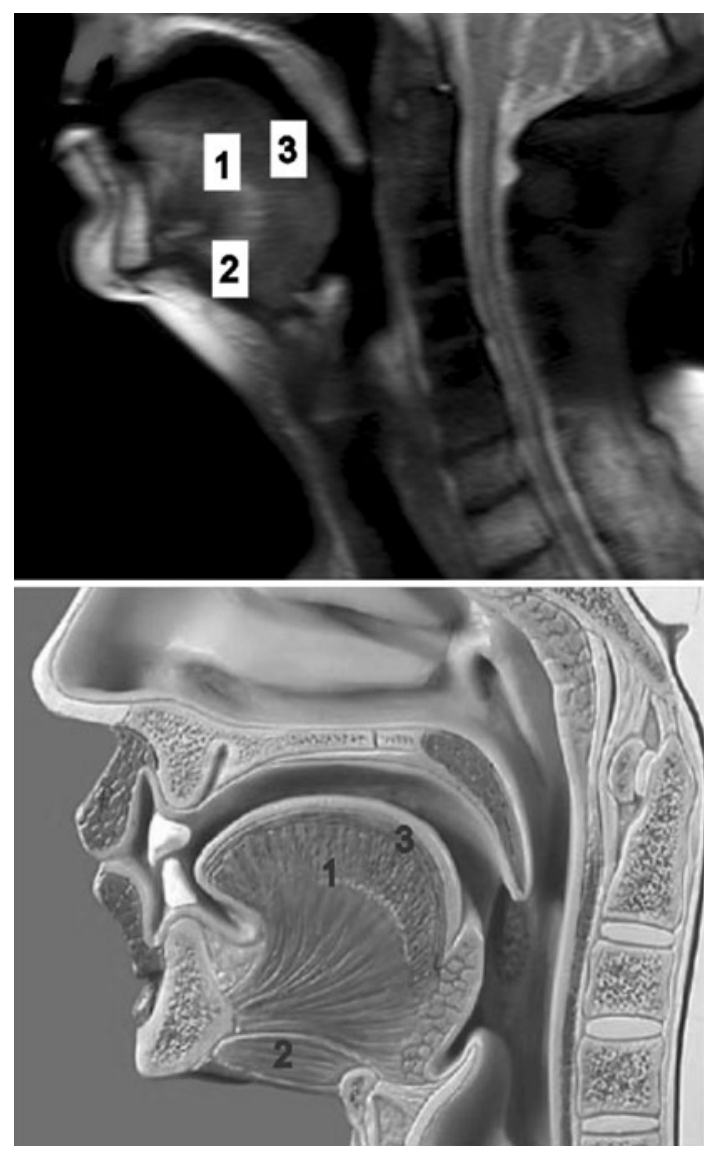

Fig. 2 Visibility of tongue muscles in cine MRI. 1 Genioglossus muscle, 2 geniohyoid muscle, 3 intrinsic tongue muscles

MRI-studies according to the criteria in Table 1 resulted in an intraobserver agreement of $90 \%$ and an interobserver agreement of $86 \%$.

Frequently, it was possible to discriminate different tongue muscles, such as the intrinsic tongue muscles, the geniohyoid and genioglossus muscle. In all high quality cine MRIs $(n=20)$, it was possible to discern the genioglossus muscle. In 19 cine MRIs, it was possible to discern the intrinsic tongue muscles; in 16, the geniohyoid muscle was visible, see Fig. 2.

Comparison of pre- and posttreatment cine MRI

Eleven patients had cine MRIs pre- and posttreatment (see Table 2 for baseline characteristics) and were available for analysis of pre- and posttreatment differences.

It was possible to evaluate differences in mobility of tongue structures and specific muscle groups in the tongue. In 9 of 11 patients, the pretreatment cine MRI immobility score was already affected due to tumor invasion and in 7 of 11 patients even less mobility was seen after therapy. Mean cine MRI immobility score pretreatment was 10.8 (range 914) and posttreatment 12.4 (range 9-17) (see Table 3). In 7 of 11 patients, the immobility score on cine MRI was higher posttreatment compared to pretreatment. In 4 of 11 patients, the score was the same. The difference between pre- and posttreatment scores is significant, $p=0.017$.

In two of the four surgical patients, the flap reconstruction could be observed, resulting in an immobile mass, the immobility depending on the size of the tissue transfer and the type of donor tissue used. In the two others, the flap was not visible as it was not in the midline.

\section{Videofluoroscopy versus cine MRI}

Mean immobility score on videofluoroscopy was also calculated; see Table 3. Quality, as evaluated by both investigators, was high in all videofluoroscopy studies. Intraobserver agreement of videofluoroscopy evaluation was $94 \%$, interobserver agreement $90 \%$.

Table 2 Patients with both a pre- and a posttreatment cine MRI

\begin{tabular}{lllllll}
\hline Pat. no. $^{\circ}$ & Sex & Age (years) & TNM stage & Tumor location & Therapy & Treatment outcome \\
\hline 1 & F & 68 & T4aN2c & Tonsil & CRT & CR \\
2 & M & 59 & T2N2b & FOM & S + RT & CRT \\
3 & M & 59 & T4bN2b & Tonsil & S & CR \\
4 & F & 77 & T2N2c & Tonsil & CRT & Postoperative status \\
5 & M & 53 & T4aN2c & Tonsil & S + RT & CR \\
6 & M & 62 & T4aN2b & BOT & CRT & CR \\
7 & M & 59 & T4aN2b & Inferior alveolar process & CRT \\
8 & M & 59 & T4aN1 & FOM & S & Postoperative status \\
9 & F & 55 & rT4aN0 & Tongue & Induction CT + CRT & Residual tumor \\
10 & M & 48 & T4aN2c & Tongue & CRT & Decrease of base of tongue tumor, \\
11 & F & 48 & T2N2c & BOT & & persisting asymmetry \\
\hline
\end{tabular}

$F$ female, $M$ male, $C R$ complete remission, FOM floor of mouth, $S$ surgery, $R T$ radiotherapy, $C T$ chemotherapy, $C R T$ concurrent chemoradiation, $B O T$ base of tongue 
Table 3 Pre- and posttreatment immobility scores of 11 patients for cine MRI and videofluoroscopy and the dysphagia score both pre- and posttreatment

\begin{tabular}{|c|c|c|c|c|c|c|}
\hline & \multicolumn{3}{|l|}{ Pretreatment } & \multicolumn{3}{|l|}{ Posttreatment } \\
\hline & $\begin{array}{l}\text { Cine MRI } \\
\text { immobility score }^{\mathrm{a}}\end{array}$ & Videofluoroscopy ${ }^{b}$ & Dysphagia $^{c}$ & $\begin{array}{l}\text { Cine MRI } \\
\text { immobility score }^{\mathrm{a}}\end{array}$ & Videofluoroscopy ${ }^{\mathrm{b}}$ & Dysphagia $^{c}$ \\
\hline 1 & 11 & $11(1)$ & 15 & 17 & $14(2)$ & - \\
\hline 2 & 9 & $9(1)$ & 18 & 9 & $12(1)$ & - \\
\hline 3 & $9(1)$ & $9(2)$ & 12 & 10 & $11(2)$ & 15 \\
\hline 4 & 11 & $10(1)$ & 17 & 12 & $13(2)$ & 23 \\
\hline 5 & 11 & $12(1)$ & 11 & 11 & $10(1)$ & 11 \\
\hline 6 & 10 & $9(1)$ & - & 12 & $12(1)$ & 19 \\
\hline 7 & 12 & $10(2)$ & 26 & $14(1)$ & $10(5)$ & 23 \\
\hline 8 & 10 & $9(1)$ & - & 10 & $9(1)$ & 19 \\
\hline 9 & 12 & $9(2)$ & - & 15 & $13(3)$ & - \\
\hline 10 & 14 & $9(3)$ & 28 & 14 & $9(1)$ & 20 \\
\hline 11 & 10 & $9(3)$ & 10 & 12 & $11(1)$ & 19 \\
\hline
\end{tabular}

The number of missed items (0-9) is shown between brackets (see Methods)

a Cine MRI immobility score, based on the criteria described in Table 1, ranging from 9 (normal)-23 (abnormal)

${ }^{\mathrm{b}}$ Videofluoroscopy immobility score, based on the criteria described in Table 1, ranging from 9 (normal)-23 (abnormal)

${ }^{c}$ Dysphagia score based on 10 swallowing items of the EORTC-H\&N 35, ranging from 10 (normal)-37 (abnormal)

In none of the videofluoroscopies, the primary tumor was visible. In 9 of 11 patients, cine MRI had extra value compared to videofluoroscopy in evaluating mobility of oral and oropharyngeal structures.

In all $(n=22)$ cine MRIs, floor of mouth mobility was visible, in 13 of 22 mobility was abnormal. In none of the videofluoroscopy images this was evaluable. Neither was it possible to evaluate mobility of the geniohyoid and genioglossus muscle nor of the intrinsic tongue muscles on videofluoroscopy as opposed to cine MRI.

Quality of life versus cine MRI and videofluoroscopy

Questionnaires were available for 22 patients and 16 questionnaires were complete. Correlation between the 10 swallowing items on the quality of life questionnaire was high, Cronbach's alpha was 0.976 , for all dysphagia scores see Table 3. Figure 3 shows the correlation between posttreatment cine MRI immobility score and posttreatment dysphagia score on quality of life questionnaire: a more abnormal cine MRI correlates with more complaints of swallowing. Spearman's correlation coefficient is $0.73(p=0.04)$. Pretreatment cine MRI immobility was not significantly correlated to pretreatment dysphagia. Spearman's correlation coefficient is $0.55(p=0.156)$.

Grouping patients with posttreatment cine MRI scores $>11.5$ and $<11.5$, this appeared to be a cutoff value: patients with more immobility on the cine MRI (a score $>11.5$ ) had significantly more dysphagia posttreatment. Patients with a

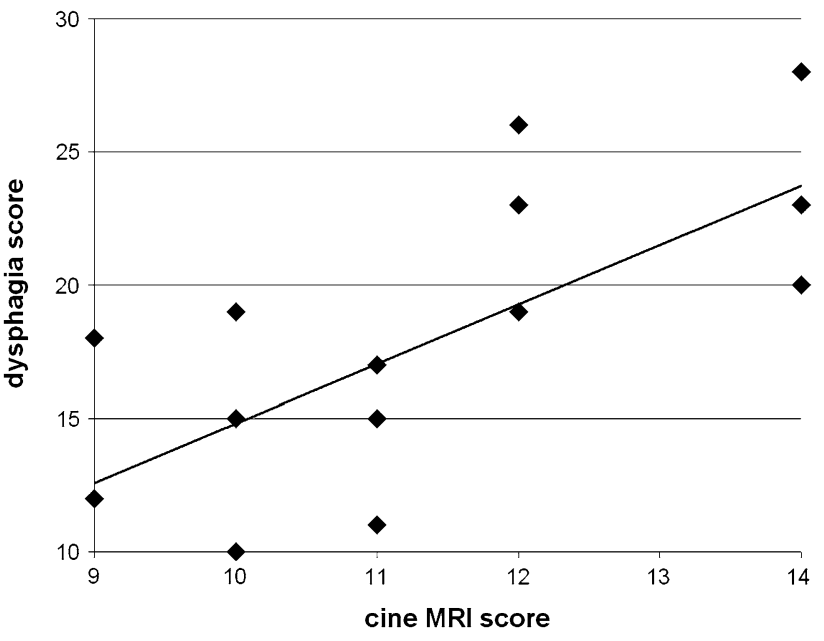

Fig. 3 Subjective dysphagia score versus cine MRI immobility score. Scatterplot presenting relation between dysphagia score (ranging from normal swallowing $=10$ until completely abnormal swallowing $=37)$ and cine MRI immobility score (ranging from normal mobility $=9$ until completely immobile $=23$ ) in 16 cine MRIs of patients with oral and oropharyngeal cancer. Both pre- and posttreatment time synchronous data were used (see Table 3). A larger dot (11-11 and 12-19) reflects two dots at the same place

cine MRI immobility score $>11.5$ had a mean dysphagia score of 21, standard deviation (SD) 2, and patients with immobility scores $<11.5$ had a mean dysphagia score 15 , $\mathrm{SD} 4, p=0.032$.

Figure 4 shows the scatter plot of videofluoroscopy immobility score and dysphagia score for all completed 


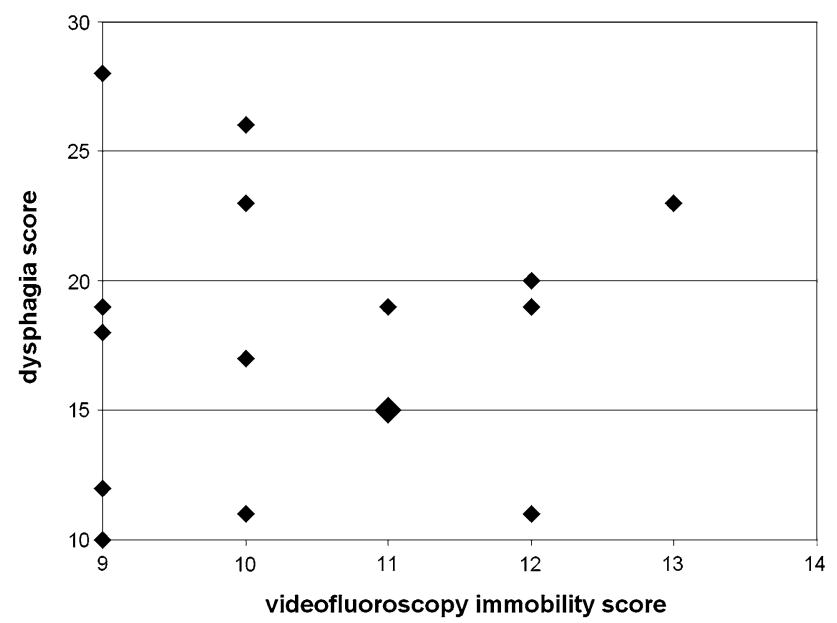

Fig. 4 Subjective dysphagia score versus videofluoroscopy immobility score. Scatterplot presenting relation between dysphagia score (ranging from normal swallowing $=10$ until completely abnormal swallowing $=37$ ) and videofluoroscopy immobility score (ranging from normal mobility $=9$ until completely immobile $=23$ ) in 16 videofluoroscopies of patients with oral and oropharyngeal cancer. Both pre- and posttreatment time synchronous data were used (see Table 3). A larger dot (11-15) reflects two dots at the same place

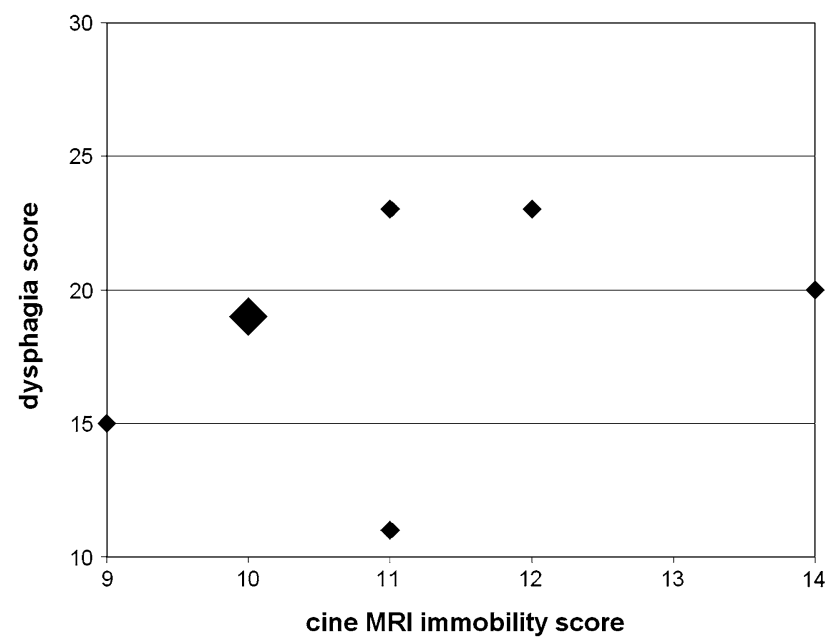

Fig. 5 Subjective dysphagia score posttreatment versus cine MRI immobility score pretreatment. Scatterplot presenting relation between posttreatment dysphagia score (ranging from normal swallowing $=10$ until completely abnormal swallowing $=37$ ) and pretreatment cine MRI immobility score (ranging from normal mobility $=9$ until completely immobile $=23$ ) in 8 patients with oral and oropharyngeal cancer. A larger dot (10-19) reflects three dots at the same place. There is no significant correlation

questionnaires. No correlation was found between the subjective swallowing and results of videofluoroscopy. Spearman correlation coefficient is $0.09, p=0.83$.

Pretreatment cine MRI score did not correlate with posttreatment dysphagia score, Fig. 5 shows a scatterplot of the 8 patients for which a posttreatment dysphagia score and a pretreatment cine MRI were available.
T-stage

Three patients had a T2 tumor; seven had a T4 tumor, and one a recurrence (rT4). We could not find significant differences neither in the questionnaires scores, nor in the immobility scores between the T2 and (r)T4 groups.

\section{Discussion}

Cine MRI of swallowing is useful to evaluate important structures for swallowing and to visualise mobility in patients with oral and oropharyngeal cancer. Cine MRI yielded additional information compared to videofluoroscopy, showing particular tongue muscles, the floor of mouth and its mobility and the tumor. Both on videofluoroscopy and cine MRI immobility of the swallowing structures increased significant posttreatment. Using our scoring system, only cine MRI was significantly correlated to the subjective swallowing problems. There was a significant correlation between an abnormal posttreatment cine MRI and posttreatment dysphagia, and there was no correlation between posttreatment videofluoroscopy and dysphagia. Moreover, we found a cutoff point, as there was a significant difference in dysphagia score between patients with an immobility score on cine MRI above 11.5 and below the cutoff of 11.5.

Additional value of preoperative cine MRI as compared to videofluoroscopy is that both tumor location and extension are visible and that these can be studied simultaneously with swallowing performance. The better visualisation of the tissues in the oral and oropharynx and the mobility (including contacts of important structures) during swallowing movement by cine MRI provides new insights in the pre- and posttreatment biomechanics of swallowing in patients with oropharyngeal carcinoma. This probably leads to a better and more detailed evaluation of swallowing pathophysiology than with standard videofluoroscopy.

The capability of cine MRI to visualize mobility of soft tissues and muscles as well as results of tissue transfer for reconstruction makes it particularly well suited for the evaluation of dysphagia in patients who have undergone surgery for advanced oral cancer [11]. Our study confirms the results of Kitano [11] who successfully analyzed cine MRI for 12 patients with dysphagia after surgery and postoperative RT for oral cancer.

As opposed to videofluoroscopy, cine MRI does not use ionizing radiation. Another advantage of cine MRI is scanning of multiple planes, e.g. axial, coronal and sagittal planes, and in case of a lateral tumor, a paramedian slice through the tumor could also be taken. Cine MRI frequently allowed for direct visualization and evaluation of 
mobility of the intrinsic tongue, geniohyoid, and genioglossus muscles. The use of one midline section, instead of the superimposed images of videofluoroscopy, potentially allows for more accurate interpretation of tumor induced mobility impairment. Cine MRI is a noninvasive technique that does not require ingestion of oral contrast medium or ingestion of a solid or liquid bolus. Since all these patients undergo a standard pretreatment MRI as part of the diagnostic work-up, additional cine MRI only takes 5 min extra. In the future, cine MRI might potentially provide new data for tailored organ preservation radiotherapy and swallowing rehabilitation or preoperative counselling of the patient with regard to expected functional outcomes. Cine MRI seems to be useful in other patient groups as well, e.g. patients with obstructive sleep apnea syndrome.

Advantages of videofluoroscopy in comparison to cine MRI are real time imaging, constant high quality images, use of a bolus in different consistencies instead of dry swallow, and a more physiologic posture. Cine MR images were sometimes affected by motion artifacts and by metallic dental material. Our study was somewhat limited by the necessary focus on items, which could be evaluated by both imaging modalities, excluding the actual swallowing movement using a bolus, confirming presence of aspiration or penetration, what can be evaluated using videofluoroscopy. Dry swallow during cine MRI does not permit evaluation of aspiration and penetration, nasal reflux or stasis. Moreover, swallowing in the supine MRI scanning position may alter compensatory mechanisms in certain dysphagic patients, who may rely in part on gravity for swallowing [10]. These advantages of videofluoroscopy make that cine MRI will not replace it, but it might be of additional value having other advantages over videofluoroscopy, as stated above.

The main weakness of the cine MRI used in our study is the low temporal resolution (1.3 images/s) and the resulting need to analyze repeated swallows [10]. However, scoring the immobility of tissue was possible without ordering the images into synchronous order. In the course of the study, we noticed that the quality of images was influenced by the velocity of swallowing. Asking patients in advance to swallow slowly gave the best image quality. Cine MRI using T1 weighted images may be performed with even better temporal resolution, but the soft tissue contrast will then be less than in T2 weighed images used in our study, thus tumor and different muscular structures are better visible in $\mathrm{T} 2$ weighed images. A shortcoming of this study is the low number of included patients and high incidence of missing values in quality of life scores. Nevertheless, we obtained new interesting significant results which need confirmation in a larger study group.

\section{Conclusion}

Cine MRI is a promising new technique as an adjunct next to standard examinations for evaluation of swallowing in patients with oral and oropharyngeal cancer as it directly visualizes the dynamics of swallowing and allows evaluation of pre- and posttreatment differences. Abnormal findings are significantly correlated with subjective swallowing complaints of patients where videofluoroscopy was unable to correlate. We are confident that cine MRI imaging opens new insights for our knowledge of swallowing dynamics in the pretreatment phase, which might help in clinical decision making and evaluation of posttreatment dysphagia. This dynamic imaging technique enabled us to evaluate the effect of treatment on swallowing, potentially providing new data for tailored organ preservation radiotherapy and swallowing rehabilitation.

Conflict of interest No conflicts of interest are to be reported.

Open Access This article is distributed under the terms of the Creative Commons Attribution Noncommercial License which permits any noncommercial use, distribution, and reproduction in any medium, provided the original author(s) and source are credited.

\section{References}

1. Morton RP (2003) Studies in the quality of life of head and neck cancer patients: results of a two-year longitudinal study and a comparative cross-sectional cross-cultural survey. Laryngoscope 113:1091-1103

2. Pauloski BR, Logemann JA, Rademaker AW et al (1994) Speech and swallowing function after oral and oropharyngeal resections: one-year follow-up. Head Neck 16:313-322

3. Ackerstaff AH, Balm AJ, Rasch CR et al (2009) First-year quality of life assessment of an intra-arterial (RADPLAT) versus intravenous chemoradiation phase III trial. Head Neck 31:77-84

4. Fuwa N, Kodaira T, Furutani K et al (2008) Treatment results of continuous intra-arterial CBDCA infusion chemotherapy in combination with radiation therapy for locally advanced tongue cancer. Oral Surg Oral Med Oral Pathol Oral Radiol Endod 105:714-719

5. Soo KC, Tan EH, Wee J et al (2005) Surgery and adjuvant radiotherapy vs concurrent chemoradiotherapy in stage III/IV nonmetastatic squamous cell head and neck cancer: a randomised comparison. Br J Cancer 93:279-286

6. Stenson KM, Kunnavakkam R, Cohen EE et al (2010) Chemoradiation for patients with advanced oral cavity cancer. Laryngoscope 120:93-99

7. Tomita N, Kodaira T, Furutani K et al (2010) Long-term followup and a detailed prognostic analysis of patients with oropharyngeal cancer treated with radiotherapy. J Cancer Res Clin Oncol 136:617-623

8. Li B, Li D, Lau DH et al (2009) Clinical-dosimetric analysis of measures of dysphagia including gastrostomy-tube dependence among head and neck cancer patients treated definitively by intensity-modulated radiotherapy with concurrent chemotherapy. Radiat Oncol 4:52

9. Hartl DM, Albiter M, Kolb F et al (2003) Morphologic parameters of normal swallowing events using single-shot fast spin echo dynamic MRI. Dysphagia 18:255-262 
10. Hartl DM, Kolb F, Bretagne E et al (2006) Cine magnetic resonance imaging with single-shot fast spin echo for evaluation of dysphagia and aspiration. Dysphagia 21:156-162

11. Kitano H, Asada Y, Hayashi K et al (2002) The evaluation of dysphagia following radical surgery for oral and pharyngeal carcinomas by cine-magnetic resonance imaging (Cine-MRI). Dysphagia $17: 187-191$
12. Rogers S, Ahad SA, Murphy AP (2007) A structured review and theme analysis of papers published on "quality of life" in head and neck cancer: 2000-2005. Oral Oncol 43:843-868

13. Bland JM, Altman DG (1997) Cronbach's alpha. BMJ 314:572 